\title{
UMA MÚLTIPLA E PROFUNDA IMERSÃO NA INFORMAÇÃO NOSSA DE CADA DIA
}

\author{
UNA MÚLTIPLE Y PROFUNDA INMERSIÓN EN LA INFORMACIÓN \\ NUESTRA DE CADA DÍA
}

GIANNASI-KAIMEN, M. J.; CARELLI, A. E. (Org.). Recursos informacionales para compartir: acceso, disponibilidad y uso. Buenos Aires: Alfagrama, 2010. 200 p. ISBN ISBN 978-987-1305-61-2

Osvaldo Nilo BalmasedaNeyra

Doctor en Ciencias Pedagógicas

Dirección de Educación de Posgrado, Ministerio de Educación Superior, Cuba Dirección Regional AUIP Centroamérica y Caribe e-mail: osbalneyra@yahoo.es

Una de las manifestaciones más perceptibles de la globalización es la necesidad de información: las decisiones del presidente de una nación pueden afectar numerosos territorios; la aparición de una enfermedad en un país cualquiera, por muy distante que se encuentre de nosotros, puede afectarnos en cuestión de horas; ningún investigador puede ejercer adecuadamente su labor si no está al tanto de la vertiginosa producción de conocimientos que propician el surgimiento de nuevos materiales o nuevas metodologías. Como consecuencia, no solo para el científico, sino para el individuo más humilde, resulta inexcusable el más actualizado conocimiento de lo que en todo momento está aconteciendo, tanto en su entorno geográfico como en la aldea global, y en ello tienen un papel fundamental la posesión de información actualizada, pertinente y confiable.

Por mucho más que lo dicho en el párrafo anterior, es digna de elogio la iniciativa de Maria Júlia Giannasi-Kaimen y Ana Esmeralda Carellial convocar a 
diez destacadas investigadoras de las más prestigiosas universidades de todo el Brasil, en el campo de la Ciencia de la Información y áreas afines,a fin de componer el libro titulado Recursos informacionales para compartir. acceso, disponibilidad y uso, lanzado en el año 2007, y preparado por el Departamento de Ciencias de la Información de la Universidad Estatal de Londrina (Universidade Estadual de Londrina), y que, ahora, gracias a la editorial argentina Alfagrama, se pone al alcance del lector hispanohablante en una cuidadosa traducción realizada por los profesores Maria das GraçasTargino y AlissonDias Gomes.

Los ocho capítulos que integran el libro abordan los más controvertidos temas relacionados con la información y la comunicación en diferentes dimensiones y desde ángulos diversos, discutiendo términos, conceptos, paradigmas, el surgimiento de nuevas tecnologías y redes académicas y sociales en el ciberespacio, lasposibilidades de acceso abierto o limitado a fuentes informacionales, así como la dicotomía publicación impresa/publicación electrónica, entre otros.

Maria das GraçasTargino abre la obra analizando el vínculo Estado y comunicación, y relacionandoel papel de los medios con los impactos de las nuevas tecnologías en la cultura contemporánea, en "Comunicación científica y Estado o Estado y comunicación científica: ¡No importa cómo se diga!” (Capítulo 1) y "Recursos tecnológicos e informacionales: la certeza de un presente virtual y la incertidumbre de un futuro de paz" (Capítulo 5), respectivamente. En ellos la eminente profesora examina las obligaciones del Estado en cuanto a la más completa apertura a los canales de distribución de la información y el derecho del ciudadano a obtener información, esta última resumida lapidariamente al decir que "[...] la información es derecho social de todos [...]"; coherentemente con esta concepción, al considerar el carácter social de la ciencia y la tecnología, reconoce que la información al actuar como el principal vehículo para la producción y difusión del conocimiento, acentúa las diferencias entre naciones e incluso entre segmentos sociales e individuos, convirtiendo las tecnologías en un factor excluyente.

Por su parte la Libre-docenteJoanaCoeli, en el Capítulo 2 "Usar o no usar la patente: ¡Ésa es la cuestión!"profundiza, entre otros temas, en el maridaje entre investigación y desarrollo, así como la importancia de la ciencia y la tecnología para la transformación económica y social de la nación. En ese sentido considera el significativo papel que desempeñan el Consejo Nacional de Desarrollo Científico y Tecnológico (CNPq) y la Coordinación de Perfeccionamiento de Personal de Nivel Superior (Capes) en la calificación del personal dedicado a la investigación científica y la innovación, y en el incremento de la calidad de la contribución científica brasileña.Como indica el título del capítulo, la autora enfatiza en la importancia de la patente como documento legal paraproteger temporalmente la propiedad intelectual sobre la creación de determinado conocimiento o producto, ya sea artístico o tecnológico y, por tanto, la vía para que el creador, inventor o innovador contribuya con la sociedad mediante la posibilidad de transferir ese conocimiento. La lectura del capítulo permite conocer la evolución histórica de la patente, el debate acerca de su empleo como indicador del desarrollo de un país y las diversas consideraciones que actualmente existen al respecto, así como la diversidad de enfoques que dan lugar a disímiles legislaciones por áreas del conocimiento y por países. 
En el Capítulo 3, Asa Fujino y DulcinéiaDilvaJacomini, al intitular su contribución como "Productos y servicios de información en la sociedad del conocimiento: de la identificación al uso" estudian el crecimiento exponencial de la información, y el apoyo que en tal sentido ofrecen herramientas y procesos para recuperar información, cada vez más sofisticados, depositada en numerosas y potentes bases de datos. Al respecto, consideran que en tal contexto, ante la avalancha informacional, el mayor desafío reside en obtener sistemas inteligentes de búsqueda, "[...] que lleven en consideración aspectos semánticos y que permitan al profesional de la información ubicar, filtrar y organizar informaciones que sean potencialmente útiles para los usuarios, pasan a ser investigados como alternativa para el caos instalado, de momento, en Internet".

“¿Creemos en Google? Dibujando de nuevo el acceso a los recursos de información" es el título del enjundioso Capítulo 4, elaborado por PatríciaZeniMarchiori quien, tras describir la arquitectura y las potencialidades del más afamado motor de búsqueda y sus aplicaciones, cuyo límite, en opinión de la autora es "el límite del usuario", analiza los diversos vaticinios, favorables y adversos que para el futuro se le pronostican a tan colosal herramienta, a la que le auguran rayar con la ciencia ficción. Mucho se enriquece el capítulo al confrontar las posibilidades para capturar información en los distintos niveles de la web: la opaca, la privada, la propietaria y la verdadera web invisible. Llama la atención la pormenorizada, exhaustiva y didáctica comparación de las posibilidades que ofrecen el Google Scholar (Google Académico) y Scirus: forScientificlnformationOnly para la búsqueda de información científicoacadémica, resaltando ventajas y limitaciones de ambos.

En notable coherencia con los capítulos precedentes,Sueli Mara Soares Pinto Ferreira ofrece el interesante capítulo 6 "Fuentes de información en tiempos de acceso abierto", en el que .discute la migración de las publicaciones impresas hacia las electrónicas como uno de los principales signos del impacto de los cambios de paradigma en la producción y comunicación del conocimiento científico en la contemporaneidad. En ese sentido, la destacada profesora aborda la tríada accesibilidad, confiabilidad y publicidaden los movimientos de Archivos Abiertos (Open Archives Initiative)y de Acceso Abierto(Open Access), surgidos ambos con el propósito de facilitar la diseminación eficiente de contenidos en Internet.Muy interesante resulta el proceso evolutivo de estos movimientos en el cual la ScientificElectronic Library Online(Scielo) ha devenido ejemplo de portal abierto entre los muchos que se citan en el capítulo. Asimismo lo es el tratamiento de los derechos de autor y la forma de evaluación de los contenidos en estos movimientos, la cualposibilita la combinación de los modos 1 y 2 de producción de conocimientos propuesto por Michel Gibbons. Por último, la autora discute las características de los diferentes repositorios conocidos, así como los software que los generan y gestionan.

En el Capítulo 7"Información e imagen: el cine y la producción de sentido y conocimiento", la profesora e investigadora Terezinha Elisabeth da Silva explica cómo el cine puede ser, como es señalado en el prólogo del libro, "una puerta abierta para la producción de conocimientos". En el texto se asume como válida la convivencia de múltiples soportes de información que provoca, al decir de la autora, "[...] un universo complejo, que sobrevive de las múltiples formas de expresión, de comunicación y de información y que exige un reconocimiento de su importancia por parte del ciudadano". En tal contexto, el cine es significativo 
recurso informacional para la sociedad contemporánea, basado en la imagen, y ello requiere "aprender a mirar de forma diferente"; aunque Terezinha Elisabeth sostiene que el cine tiene poderes incontestables, que van mucho más allá de la representación al poner en función todos nuestros sentidos. El análisis discurre sobre las diferencias entre el código lingüístico y el de la imagen en movimiento.

El Capítulo 8, "Biblioteca digital y desarrollo de la competencia informacional: recursos y habilidades indispensables en la educación a distancia", elaborado por Maria Júlia Giannasi-Kaimen, Ana Esmeralda Carelli y Vilma Aparecida Gimenes da Cruz, cierran esta valiosa obra colectiva, y está dedicado a examinar las habilidades que ha de poseer el estudiante de la modalidad a distancia, dadas las particularidades del proceso de enseñanza-aprendizaje, basado en recursos tecnológicos para la distribución de información que requieren de "[...] facilidades de comunicación interactiva entre el formador y el aprendiz". Las autoras discuten y esclarecen definiciones y conceptos de esta modalidad pedagógica, deteniéndose en la educación on-line, estableciendo no solo diferencias y semejanzas entre el aula virtual y el aula presencial, sino argumentando la posiblesuperioridad de la primera sobre la segunda en materia afectiva y desarrollo de la independencia cognitiva del estudiante. Al respecto, las autoras destacan el papel que desempeña la biblioteca digital para brindar y obtener información pertinente y confiable, para lo cual deben formarse las correspondientes competencias informacionales, no solo en los estudiantes, sino también en los profesores y los bibliotecarios. Muy provechosa, desde el punto de vista didáctico, resulta la descripción de los patrones de desempeño e indicadores de las competencias informacionales con la que se cierra el capítulo.

Por último, resta agradecer a la editorial Alfragrama esta admirable idea de ofrecer al público hispanoamericano la posibilidad de conocer cuánto camino ha recorrido Brasil en materia de información y comunicación; qué sucede al respecto actualmente en el mundo y especialmente en ese subcontinente; y qué discuten hoy y cuáles son los principales desafíos para los investigadores a nivel mundial $\mathrm{y}$, en particular, los brasileños más avezados en esas áreas. Ello lo convierte en referencia obligada para todo investigador que desee explorar entre las luces y las sombras de los insondables caminos de la información y la comunicación. 